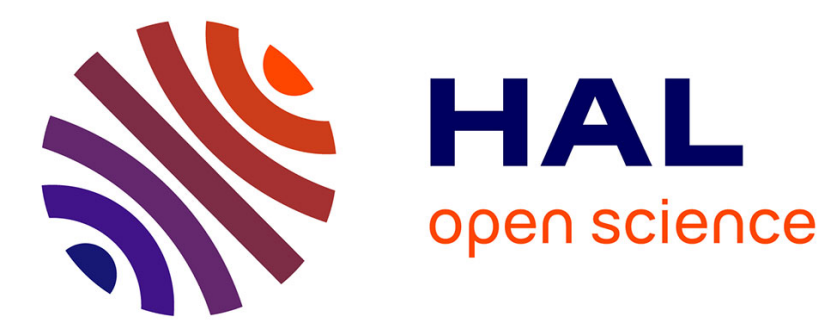

\title{
Application à un convertisseur continu-alternatif complexe d'une méthode systématique de synthèse
}

A. Courteix, Y. Cheron, H. Foch, M. Metz

\section{To cite this version:}

A. Courteix, Y. Cheron, H. Foch, M. Metz. Application à un convertisseur continu-alternatif complexe d'une méthode systématique de synthèse. Revue de Physique Appliquée, 1986, 21 (6), pp.357-364. 10.1051/rphysap:01986002106035700 . jpa-00245454

\section{HAL Id: jpa-00245454 https://hal.science/jpa-00245454}

Submitted on 1 Jan 1986

HAL is a multi-disciplinary open access archive for the deposit and dissemination of scientific research documents, whether they are published or not. The documents may come from teaching and research institutions in France or abroad, or from public or private research centers.
L'archive ouverte pluridisciplinaire HAL, est destinée au dépôt et à la diffusion de documents scientifiques de niveau recherche, publiés ou non, émanant des établissements d'enseignement et de recherche français ou étrangers, des laboratoires publics ou privés. 


\title{
Application à un convertisseur continu-alternatif complexe d'une méthode systématique de synthèse
}

\author{
A. Courteix, Y. Cheron, H. Foch et M. Metz. \\ I.N.P.T.-ENSEEIHT, Laboratoire d'Electrotechnique et d'Electronique Industrielle, \\ Unité associée au C.N.R.S. no 847, 2, rue Camichel, 31071 Toulouse Cedex, France
}

(Reçu le 14 janvier 1986, révisé le 25 février, accepté le 25 février 1986)

\begin{abstract}
Résumé. - Les auteurs se proposent de déterminer la structure d'un convertisseur complexe de façon aussi systématique que possible. Dans ce but ils définissent des lois d'association de convertisseurs élémentaires. L'application de ces lois, et le respect d'un cahier des charges, permettent non seulement de proposer des solutions originales et intéressantes, mais aussi d'envisager la généralisation de cette méthode. Une réalisation pratique valide la démarche adoptée.
\end{abstract}

\begin{abstract}
The authors want to build complex converter as systematically as possible. So, some rules for the association of elementary converters were defined. The application of these laws and the observation of specifications permit to get some original and interesting solutions. Moreover, experimental results validate the chosen way and mean that the generalization for any complex converters may be considered.
\end{abstract}

\section{Introduction.}

La recherche de structures de convertisseurs statiques adaptées aux problèmes spécifiques à résoudre a suscité de nombreuses études tant dans les domaines de pointe que dans les domaines industriels [1-5].

C'est pourquoi la structure des convertisseurs devient de plus en plus le fruit d'un raisonnement construit.

Les études actuelles mènent généralement à un meilleur compromis entre la simplicité de la structure et d'autres critères tels que la puissance massique, le rendement, le rayonnement..., etc... Elles remettent en cause des structures parfois trop figées au profit de montages plus sophistiqués et plus performants.

L'objet de cet article est d'étudier les convertisseurs permettant de réaliser à partir d'une source basse tension continue, une source haute tension alternative basse fréquence. Ce générateur de tension sinusoïdale est en particulier très recherché dans le cadre de l'énergie solaire $[4,5]$, des groupes de secours, ou du matériel embarqué. La plupart des applications de ces domaines nécessitent un système très fiable et souvent une sortie à faible taux d'harmoniques. C'est pour cette raison qu'il est important d'avoir un éventail de solutions afin de pouvoir choisir une structure bien adaptée au type de conversion désirée.
Pour résoudre ce problème complexe, les auteurs se proposent de travailler de façon très systématique.

Après avoir donné les lois générales d'association des convertisseurs élémentaires, les auteurs analysent le cahier des charges, et dégagent une ébauche des structures recherchées (éléments constitutifs, caractéristiques d'entrée et de sortie).

Puis en fixant le nombre de conversions élémentaires ils obtiennent toutes les solutions envisageables en fonction de la position et de la nature des éléments passifs.

Leur démarche est validée par l'étude expérimentale d'une solution originale.

\section{Lois d'association des convertisseurs élémentaires.}

2.1 DéFINITIONS. - Un convertisseur étémentaire est un ensemble constitué uniquement d'interrupteurs permettant de contrôler les échanges d'énergie entre deux branches électriques caractérisées par leur impédance instantanée.

Une branche est dite branche tension (resp. courant) lorsque son impédance instantanée tend vers zéro (resp. l'infini).

Nous rappelons que l'impédance instantanée est la limite de l'impédance symbolique lorsque la variable symbolique tend vers l'infini. 
2.2 Lor 1. - Un convertisseur élémentaire ne peut lier que deux branches de nature différente [1].

Un ensemble composé d'une branche tension (resp. courant) et d'un élément à caractère inductif en série (resp. capacitif en parallèle) se comporte comme une branche courant (resp. tension).

Donc il vient :

2.3 Lor 2. - L'association d'un convertisseur élémentaire et d'un élément passif bien choisi permet de lier deux branches de même nature.

Une conséquence des lois 1 et 2 est la suivante :

2. 4 Lor 3. - Sauf cas particulier $\left({ }^{1}\right)$, il est possible de décomposer tout convertisseur en une association de convertisseurs élémentaires et d'éléments passifs.

2.5 Lor 4. - Le choix de la place et de la nature des éléments passifs fixe la nature des branches d'entrée (donc des branches de sortie (Loi 1)) de tous les convertisseurs élémentaires de l'association.

\subsection{LoI 5. - (Schéma no 1$)$}

A) Réversibilité en courant :

La mise en série d'une branche tension avec un élément à caractère inductif donne une branche courant (Loi 2) possédant la même réversibilité en courant que la branche tension.

\section{B) Réversibilité en ten-} sion:

L'inductance joue un rôle de tampon au niveau des tensions : $V_{\text {moyen }}=V_{\text {moyen }}^{\prime}$ alors que

$$
V_{\text {instantané }} \neq V_{\text {instantane }}^{\prime} \text {. }
$$

En conséquence la branche résultant de la mise en série d'une branche tension avec un élé-

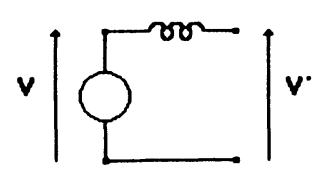

Schéma 1. - Notations.

[Notations].

(1) Convertisseurs à accumulation inductive ou capacitive. ment à caractère inductif a une réversibilité potentielle en tension.

Cette réversibilité n'est pas toujours utilisée.

Dans certains cas partiliers, il est possible de conclure sur la réversibilité de $V^{\prime}$ : En effet si $V_{\text {moyen }}=0$ et $V^{\prime}$ pas toujours nul, alors $V^{\prime}$ est réversible en tension.

\section{Analyse du cahier des charges et conséquences.}

- Il s'agit de réaliser à partir d'une basse tension continue (batterie $24 \mathrm{~V}$ par exemple) un générateur de tension sinusoïdale basse fréquence $(220 \mathrm{~V}, 50 \mathrm{~Hz}$ par exemple), capable de débiter sur une charge complexe (dont certains éléments sont réactifs), grâce à un convertisseur statique autonome.

Dans la suite de l'exposé, les tensions côté sortie, dont la valeur est élevée par rapport à la tension d'entrée, seront appelées hautes tensions.

Il existe un grand rapport entre la tension d'entrée et la tension de sortie du convertisseur qui impose d'utiliser un transformateur, lequel donne de plus l'isolement galvanique.

Le cahier des charges impose à ce convertisseur de se comporter vu de la sortie comme une source de tension alternative basse fréquence. En conséquence la sortie sera filtrée par un condensateur en parallèle avec la charge.

D'après le cahier des charges, l'entrée est une branche tension unidirectionnelle en tension. D'après le paragraphe précédent, l'ensemble condensateur plus charge est une branche tension bidirectionnelle en tension et courant. Donc l'entrée et la sortie du convertisseur étant des branches de même nature (tension), d'après la loi 2, nous trouverons un élément à caractère inductif (inductance ou circuit $L-C$ série ou plus généralement un circuit comportant une inductance en série) dans le convertisseur.

A partir des résultats précédents, il est possible, à condition de définir le nombre maximum d'éléments, d'établir une classification des convertisseurs réalisant l'échange d'énergie souhaité selon des critères à définir (nombre de convertisseurs élémentaires en série, réversibilité en puissance instantanée des convertisseurs élémentaires, présence et niveau d'un élément de liaison ou de stockage, ..., etc...). Cependant une telle classification, serait trop développée et comporterait de nombreuses structures inutilisables. Cest pourquoi, nous allons chercher à réduire celle-ci en affinant le cahier des charges.

- La recherche de performances élevées nous mène à une structure fonctionnant le plus possible en haute tension pour améliorer le rendement et travaillant en haute fréquence pour augmenter la puissance massique. 
Le transformateur et les éléments passifs bénéficieront de la réduction de taille due à la haute fréquence.

En raison de sa haute fréquence de fonctionnement, le transformateur ne peut être relié directement ni à la sortie qui est une tension basse fréquence, ni à l'entrée qui est une tension continue. Il devra être situé entre deux convertisseurs élémentaires.

On en déduit que le convertisseur sera une association d'au moins deux convertisseurs élémentaires.

De plus pour des raisons de rendement il faut travailler le moins possible en basse tension : si le convertisseur est composé de plus de deux convertisseurs élémentaires le transformateur sera situé le plus près possible de la source.

Pour contrôler le courant magnétisant, la valeur moyenne de la tension à l'entrée d'un transformateur doit être nulle. Ainsi pour que le transformateur travaille en haute fréquence les harmoniques de tension basse fréquence doivent être nuls (ou négligeables $\left({ }^{2}\right)$ ) : la tension primaire sera donc une tension alternative haute fréquence [6]. C'est-à-dire une tension dont la valeur moyenne est nulle sur une période de la haute fréquence.

Par ailleurs, sachant que seuls les harmoniques (de la tension et du courant) de même rang peuvent fournir une puissance moyenne non nulle, nous déduisons du paragraphe précédant que les harmoniques basse fréquence du courant ne transmettent pas de puissance. En particulier si le courant d'entrée (ou de sortie) du transformateur est unidirectionnel, il possède une composante continue qui est source d'un surdimensionnement en courant crête des semi-conducteurs et des éléments passifs, donc d'une augmentation notable des pertes d'autant plus importante que l'on travaille en basse tension. Nous considérerons donc que seules les solutions utilisant des courants bidirectionnels à valeur moyenne nulle et dont les harmoniques basse fréquence sont négligeables permettent d'optimiser la structure de notre convertisseur [6], bien que dans certains cas la bidirectionnalité du courant augmente la complexité de la structure du convertisseur.

La plupart des charges alternatives sont des charges complexes. Ceci implique l'existence d'une puissance instantanée alternative au niveau de la charge alors que la puissance moyenne peut rester positive.

Dans la phase où la puissance instantanée est négative la charge renvoie momentanément de l'énergie vers l'entrée du convertisseur. Celle-ci peut :

- transiter intégralement jusqu'à la source

- être stockée, à la sortie du convertisseur ou à un niveau intermédiaire, par un étage tampon, composé d'éléments à caractère inductif ou capacitif ou d'une

${ }^{(2)}$ Les composantes de tension basse fréquence si elles existent doivent créer une variation du courant magnétisant négligeable devant les valeurs de courant magnétisant dues à la haute fréquence. batterie, capable d'absorber cette énergie restituée par la charge.

Les éléments passifs sont des éléments haute fréquence et ne peuvent donc stocker l'énergie basse fréquence restituée par la charge. La puissance instantanée négative transitera intégralement de la sortie vers l'entrée du convertisseur.

C'est pourquoi le convertisseur sera totalement réversible en puissance instantanée. Tous les convertisseurs élémentaires de l'association seront donc réversibles en puissance instantanée.

De plus, la puissance instantanée négative transitant jusqu'à la source (entrée du convertisseur), cette dernière doit être réversible en puissance instantanée donc bidirectionnelle en courant puisqu'il s'agit d'une source de tension continue.

- En définitive le convertisseur recherché est composé d'au moins deux convertisseurs élémentaires réversibles en puissance instantanée et d'un élément à caractère inductif. Il a pour entrée une branche tension bidirectionnelle en courant, et pour sortie une branche bidirectionnelle en tension et courant. Son isolement galvanique est situé après le premier convertisseur élémentaire et voit des tensions et courants alternatifs haute fréquence (Schéma no 2).

\section{Synthèse.}

4.1 AsSOCIATION DE DEUX CONVERTISSEURS ÉLÉMENTAIRES. - La synthèse des convertisseurs passe par la détermination de la nature et des réversibilités des branches d'entrée et de sortie de tous les convertisseurs élémentaires composant l'association.

Nous considérerons dans la suite de l'exposé que les dipôles d'entrée ou de sortie d'un convertisseur élémentaire sont assimilables à des branches, quelle que soit leur composition (association d'éléments à caractère inductif ou capacitif, de convertisseurs élémentaires, et de sources et de charges).

- Le fait de choisir la place de l'élément à caractère inductif fixe la nature des branches d'entrée et de sortie des deux convertisseurs élémentaires (Loi 4, § 2).

Les entrées des convertisseurs élémentaires situés en amont (resp. : en aval) de l'élément à caractère inductif sont des branches tension (resp. : courant) (Schéma no 3).

La détermination de la nature des branches d'entrée fixe la nature des branches de sortie car un convertisseur élémentaire lie deux branches de nature différente (Loi 1, § 2).

Tout ceci reste vrai quel que soit le nombre des convertisseurs élémentaires composant l'association tant que la nature d'une branche n'est pas changée par l'adjonction d'un condensateur en parallèle.

- Les réversibilités en tension et en courant au niveau de l'entrée et de la sortie du convertisseur, et du transformateur ont été totalement définies au para- 


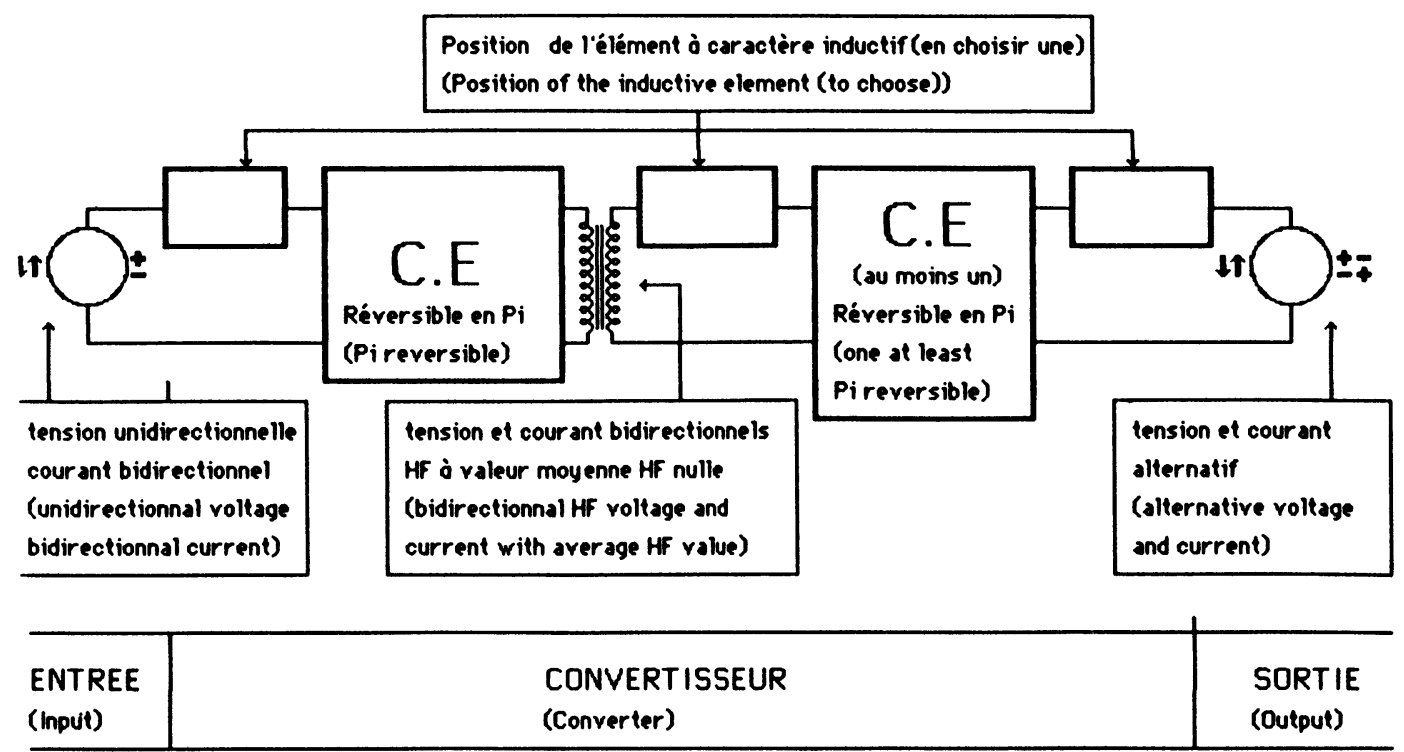

C.E. : Convertisseur élémentaire (Elementary converter)

$\mathrm{Pi}$ : Puissance instantanée (Instantaneous power)

Schéma 2. - Ebauche de la structure du convertisseur.

[Sketch of the converter structure.]

graphe 3 (Schéma no 2) :

- L'entrée du convertisseur est non réversible en tension, réversible en courant.

- La sortie du convertisseur est réversible en courant et en tension.

- La tension et le courant primaire du transformateur sont alternatifs.

En conséquence les réversibilité en courant et ten- sion, d'entrée ou de sortie, de certains convertisseurs élémentaires sont encore non définies (Flèches sur le Schéma no 3).

D'après la loi $5 \mathrm{~A}$, l'élément à caractère inductif, étant en série avec une branche tension réversible en courant, les branches (courant) résultantes sont réversibles en courant.

Dans les cas II et III (Schéma $\left.n^{\circ} 3\right)$ : nous nous
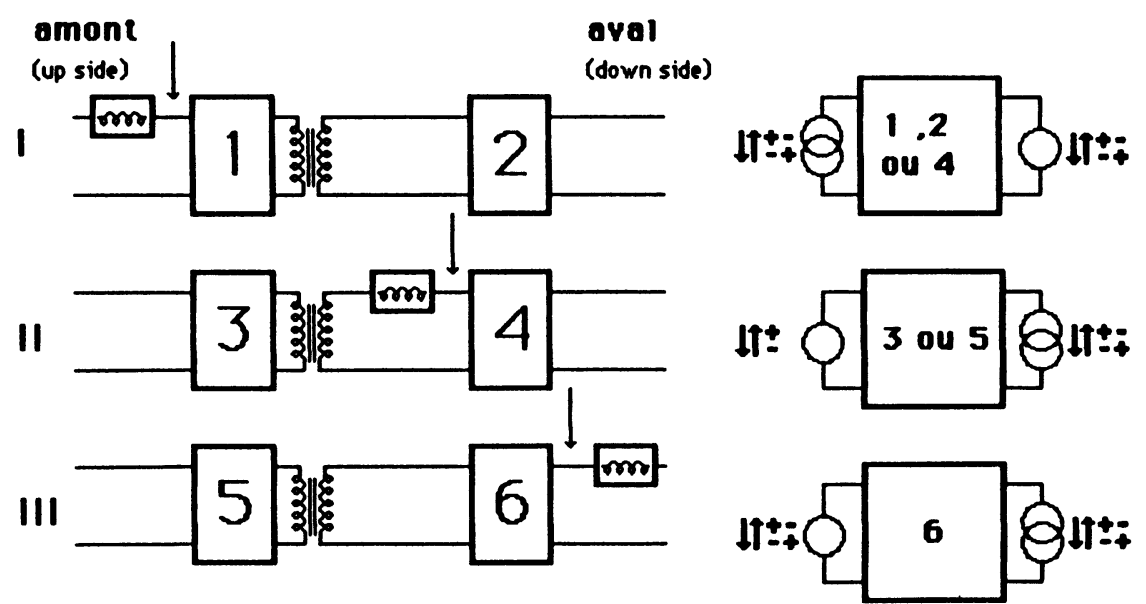

Changeur de

iréquence

courant/tension

(Frequency changer current/roltage)

Onduleur de tension (Voltoge inverter)

Changeur de fréquence

tension/courant (Frequency changer voltage/current)

\Position des branches dont les réversibilités sont ò déterminer (Position of the branches of wich reversibility are undefined)

brrs Elément à caractère inductif (Inductive element)

Schéma 3. - Résultats de la synthèse.

[Synthesis results.] 
retrouvons dans les conditions du cas particulier exposé dans la loi 5B, les branches (courant) résultantes sont réversibles en tension.

Dans le cas I (Schéma $\left.n^{\circ} 3\right)$ : si on n'utilise pas la réversibilité en tension de la branche courant (résultant de la mise en série de l'élément à caractère inductif et de la branche tension) le convertisseur élémentaire en aval de cette branche sera un convertisseur asymétrique dont les lois de commande seront complexes car il sera nécessaire de le piloter en fonction du signe de sa tension de sortie. Cette méthode de commande entraîne une lourdeur pénalisante au niveau du contrôle des transferts d'énergie (Loi 5).

Par contre si on utilise la réversibilité potentielle en tension de cette branche, le convertisseur élémentaire en aval de cette branche sera un convertisseur symétrique dont les lois de commande seront plus simples.

C'est pourquoi nous considérerons que cette branche est réversible en tension.

En résumé seules les branches d'entrée du convertisseur élémentaire le plus en amont dans les cas II et III (Schéma $n^{\circ} 3$ ) sont réversibles en courant, unidirectionnelles en tension.

Toutes les autres branches (d'entrée ou de sortie) sont réversibles en courant et tension.

- Désormais nous connaissons la nature et les réversibilités des entrées et sorties de chacun des convertisseurs élémentaires composant l'association. Il est donc possible de les identifier (Schéma no 3).

Les solutions II et III élaborent simplement la haute fréquence nécessaire au transformateur par l'onduleur de tension puis utilisent une conversion plus complexe type changeur de fréquence.

La solution I est plus complexe à commander que les autres solutions car elle est composée de deux changeurs de fréquence donc d'un nombre d'éléments commandables plus important.

C'est pourquoi dans la suite de l'exposé la solution I sera délaissée au profit des solutions II et III.
La solution II est en cours d'étude et fera l'objet d'autres publications, c'est pourquoi nous ne nous intéresserons dans la suite de l'exposé qu'à la solution III.

4.2 SOLUTIONS À TROIS ÉTAGES. - La solution retenue (solution III) reste complexe, car elle est composée d'un changeur de fréquence, c'est-à-dire (Schéma no 3) d'un convertisseur élémentaire dont les quatre interrupteurs sont bidirectionnels en courant et en tension. La commande d'un tel convertisseur est délicate et mérite une attention toute particulière afin d'éviter le court-circuit de la branche tension ou le circuit ouvert de la branche courant.

Cest pourquoi, afin de réduire les inconvénients précédemment évoqués, nous allons rechercher des structures réalisant plus simplement la fonction changeur de fréquence. Il est possible en effet d'obtenir cette fonction par une conversion indirecte, c'est-à-dire en utilisant un ou des étages intermédiaires continus. L'étage intermédiaire continu doit être une branche courant afin de permettre les échanges d'énergie entre deux sources de tension alternatives. Il existe deux types de convertisseurs indirects réversibles en puissance instantanée qui ont la fonction demandée :

- Les montages à circulation de courant (Schéma no 4 (b)).

- L'association redresseur-commutateur (Schéma no 4 (c)).

De plus si on réalise dans la solution (c) (Schéma $n^{\circ}$ 4) une modulation basse fréquence de la tension à la sortie du redresseur, il est possible d'obtenir des arches de courant basse fréquence dans l'élément à caractère inductif, ce qui simplifie la commande du commutateur car ses interrupteurs travaillent alors en basse fréquence.

Par contre dans la solution (b) (Schéma no 4) tous les interrupteurs fonctionnent en haute fréquence.

C'est pourquoi nous ne nous intéresserons dans la suite qu'à la solution (c), composée successivement

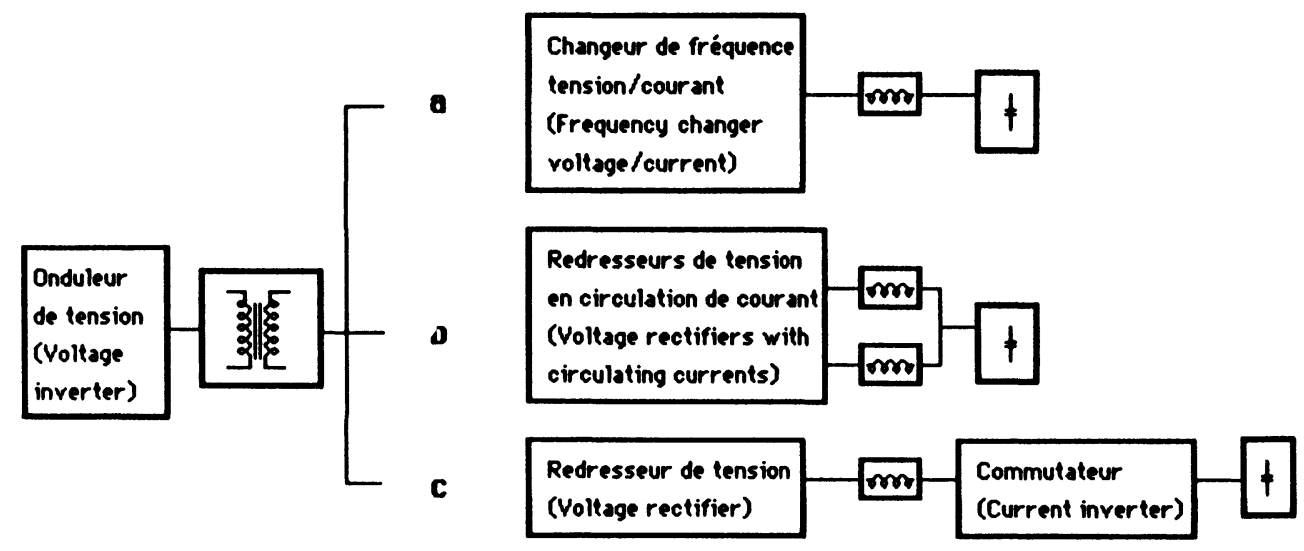

Schéma 4. - Structures déduites de la solution III.

[Solution III deduced structures.] 
d'un onduleur de tension, d'un transformateur, d'un redresseur de tension, d'un élément à caractère inductif et d'un commutateur.

\section{Exemple d'application. Résultats expérimentaux}

5.1 Evolution De la STRUCtURe ObTenUe. - Nous connaissons désormais les caractéristiques statiques de tous les interrupteurs [1]. Nous allons remplacer ceux-ci par des semi-conducteurs permettant d'optimiser les commutations.

Nous recherchons tout particulièrement à obtenir des commutations naturelles, gages de sécurité accrue et de pertes par commutation diminuées.

Cest pour cette raison, que nous avons choisi un déphasage relatif entre les commandes des bras de l'onduleur et du redresseur qui impose d'utiliser des thyristors duaux pour l'onduleur et des thyristors pour le redresseur.

Pour s'affranchir des problèmes liés au courant magnétisant le transformateur est alimenté par un créneau de tension haute fréquence fixe et de rapport cyclique un demi. Le deuxième étage impose aux interrupteurs du premier étage, une conduction des diodes précédant celle du transistor. Le blocage du courant dans une diagonale amène l'amorçage spontané (au zéro de tension) des interrupteurs de l'autre diagonale. Donc le premier étage est un onduleur de tension à fréquence fixe et à thyristors duaux (Schéma no 5).

Le deuxième convertisseur élémentaire est alimenté par une tension alternative et débite sur une charge courant constante à l'échelle de la haute fréquence. Il est classique d'utiliser pour ce genre de conversion un redresseur de tension à thyristors commandé de façon à bénéficier de la commutation naturelle (Schéma $n^{0} 5$ ).
Le temps de recouvrement inverse des thyristors impose une valeur limite à la fréquence de fonctionnement (haute fréquence). Pour une bonne fiabilité celle-ci ne doit pas être supérieure à une dizaine de kilohertz. C'est pourquoi ce convertisseur travaille à $10 \mathrm{kHz}$.

Il est important de noter que le redresseur fonctionne en haute fréquence ce qui est assez peu habituel.

Le troisième étage permute une arche sur deux de courant, il débite sur une charge complexe dont le facteur de puissance est inconnu. Les commutations seront donc naturelles ou forcées : pour troisième étage nous avons choisi un commutateur de courant à thyristors fonctionnant en commutation forcée et commutant au zéro de courant (Schéma no ${ }^{\circ}$ ).

Nous constatons que tous les interrupteurs travaillant en haute fréquence ont des commutations naturelles.

5.2 CONTRÔLE DU TRANSFERT DE PUISSANCE. - L'onduleur travaillant à fréquence fixe $(\$ 5.1)$, le plus simple est de déphaser la commande des bras du redresseur par rapport à celle des bras de l'onduleur d'un angle variant selon une loi basse fréquence.

De plus, des études théoriques et des simulations ont mis en évidence que la relation entre le déphasage des deux ponts et la valeur de la tension est pratiquement linéaire, ce qui permet une loi de commande simple.

5.3 Résultats EXPÉRIMENTAUX. - Il a été réalisé une maquette conforme au schéma ${ }^{\circ} 5$ qui a permis de prouver l'intérêt pratique de la méthode de synthèse proposée en l'illustrant par quelques formes d'ondes expérimentales, démontrant de plus la faisabilité de la structure obtenue (Schéma ${ }^{\circ}{ }^{6}$ ).

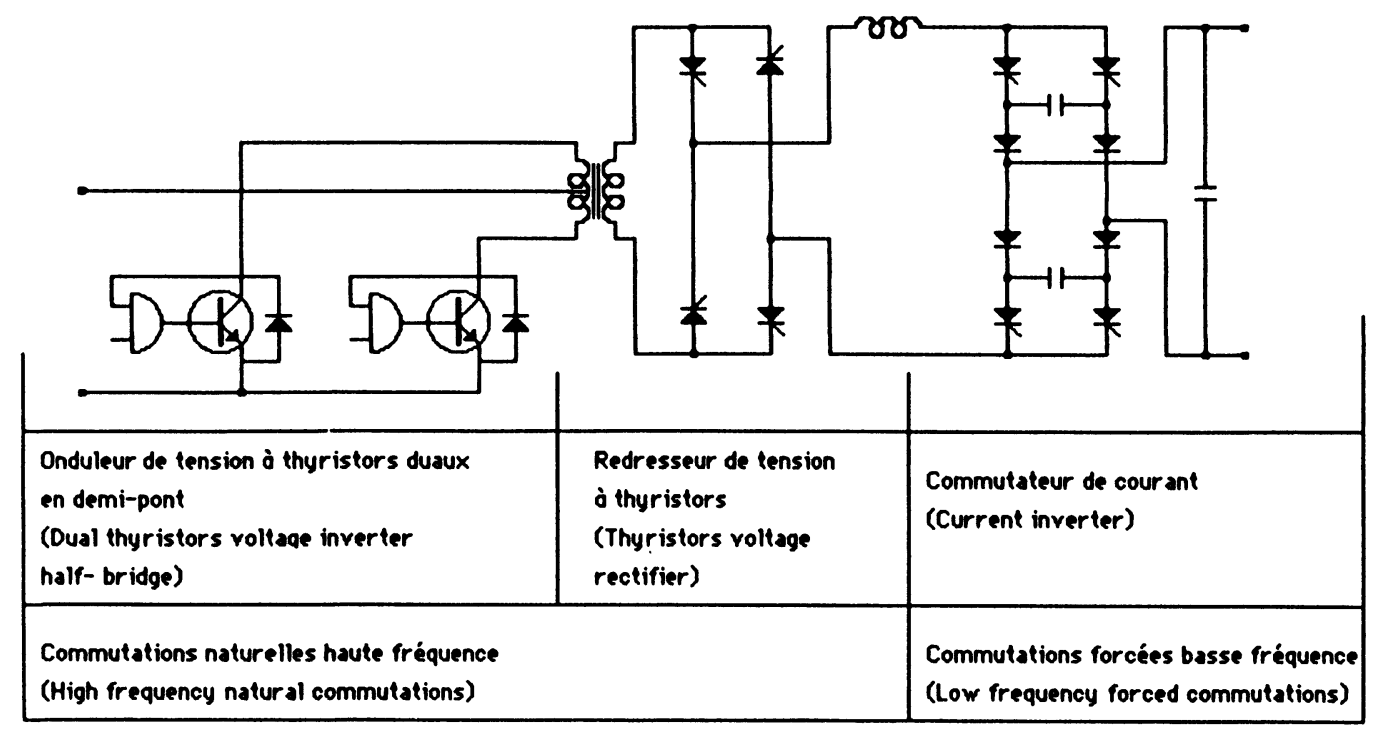

Schéma 5. - Structure du convertisseur étudié.

[Studied converter structure.] 

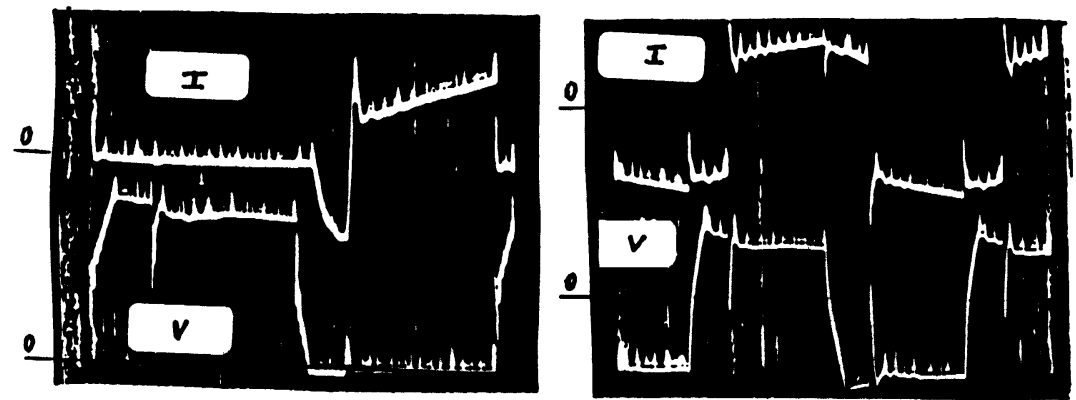

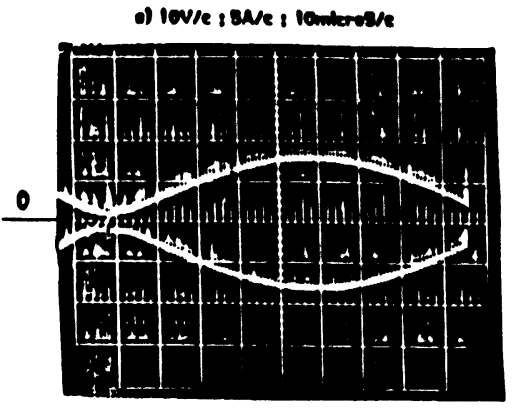

e) oswe inse

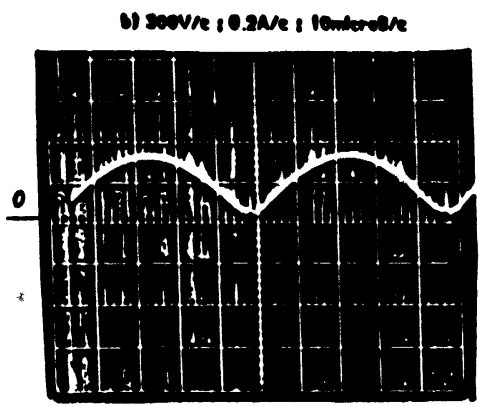

o osare inate

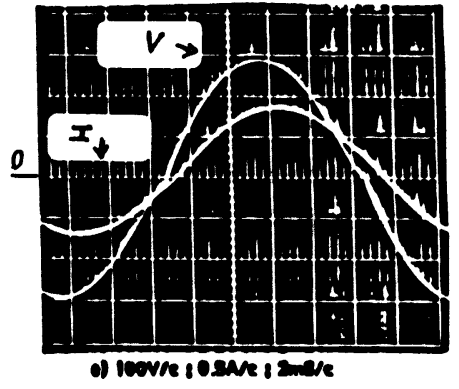

o) reovre iosure isme

Schéma 6. - Formes d'onde expérimentales pour le montage (c), fréquence de découpage $10 \mathrm{kHz}$, fréquence de sortie du convertisseur $50 \mathrm{kHz}$. a) Courant et tension (convention récepteur) à l'échelle de la haute fréquence dans un interrupteur du premier étage. b) Courant et tension (convention récepteur) à l'échelle de la haute fréquence au secondaire du transformateur. c) Courant modulé au secondaire du transformateur. d) Courant dans l'inductance (située entre le deuxième et le troisième convertisseur élémentaire). e) Courant et tension au niveau de la charge.

[Experimental waveforms for the structure (c), switching frequency $10 \mathrm{kHz}$, output frequency $50 \mathrm{kHz}$. a) High frequency current and voltage (receiver convention) in a switch of the tension inverter. b) High frequency current and voltage (receiver convention) at the output of the transformer. c) Modulated current at the output of the transformer. d) Current in the inductor (placed between the tension rectifier and the current inverter). e) Load current.]

Les résultats obtenus montrent qu'une structure relativement complexe (trois étages) permet d'obtenir un rendement et une puissance massique plus importantes que dans les solutions classiques à modulation de largeur d'impulsion ou utilisant des transistors fonctionnant en linéaire, et présente l'avantage de pouvoir délivrer une tension de sortie sur une large plage de fréquence : du continu jusqu'à la fréquence de coupure du filtre de sortie.

\section{Conclusion.}

La démarche adoptée est une démarche généralisable à l'étude de tout convertisseur complexe.

En effet il est possible de compléter les recherches effectuées précédemment sur la synthèse automatique des convertisseurs élémentaires [1] et d'étendre celles-ci à un ensemble de convertisseurs élémentaires et d'éléments passifs.

Le choix du nombre de convertisseurs élémentaires et la connaissance de la nature des entrées et sorties du convertisseur permettent de définir le nombre et la nature des éléments passifs (il existe plusieurs solutions).

A ce stade, pour chaque solution, le choix de la position des éléments passifs (et de leur nature) déterminent la nature et les réversibilités des branches d'entrée et de sortie de chacun des convertisseurs élémentaires de l'association, et donc permettent d'en effectuer la synthèse [1].

La synthèse des solutions envisageables est alors terminée car les éléments de l'association sont connus et placés.

Cependant il est intéressant dans la plupart des cas de réduire l'éventail des solutions le plus tôt possible, par l'observation d'un cahier des charges et/ou de critères physiques.

En définitive, cette méthode, systématique et reproductible, permettra certainement, une fois bien développée, une approche rapide et complète de tout les convertisseurs complexes. 


\section{Bibliographie}

[1] MoPTy, Y., Méthode de synthèse automatique des convertisseurs statiques, application à la recherche de nouveaux convertisseurs. Thèse de docteur ingénieur, Toulouse (1982).

[2] Cheron, Y., Application des règles de la dualité à la conception de nouveaux convertisseurs à transistors de puissance, Synthèse du thyristor dual, Domaine d'application. Thèse de docteur ingénieur, Toulouse (1982).

[3] Trzinsky, P., Etude et réalisation d'un convertisseur continu-alternatif à modulation de largeur d'impulsion. Thèse de docteur ingénieur, Orsay (1980).

[4] Steigerwald, R. L., Ferraro, A., Turnbull, F. G., Application of power transistors to residential and intermediate rating photovoltaic array power conditionners, IEEE Trans. Ind. Appl. IA 19 no 2 (1983).
[5] Cocconi, A., Cuk, S., Middlebroock, R. D., High frequency isolated $4 \mathrm{~kW}$ photovoltaic inverter for utility interface. PCI/MOTOR CON (1983).

[6] Steigerwald, R. L., Tompkins, R. E., A comparison of high frequency link schemes for interfacing a DC source to utility grid, IEEE Trans. Ind. Appl. IAS (1982).

[7] THOMSON CSF, Division semi-conducteurs, Le transsistor de puissance dans son environnement (1978).

[8] THOMSON CSF, Division semi-conducteurs, Le transistor de puissance dans la conversion d'énergie (1983).

[9] Cours Polycopié ENSEEIHT. Hacheurs et onduleurs autonomes. Toulouse (Edition 1983). 